Article

\title{
Analysis and Optimized Design of Metamaterials for Mid-Range Wireless Power Transfer Using a Class-E RF Power Amplifier
}

\author{
Cancan Rong ${ }^{1}{ }^{\mathbb{D}}$, Xiong Tao ${ }^{1}$, Conghui Lu ${ }^{2}$, Zhaoyang $\mathrm{Hu}^{1}{ }^{1}$, Xiutao Huang ${ }^{1}{ }^{\circledR}$, Yingqin Zeng ${ }^{1}$ \\ and Minghai Liu ${ }^{1, *}$ \\ 1 State Key Laboratory of Advanced Electromagnetic Engineering and Technology, School of Electrical and \\ Electronic Engineering, Huazhong University of Science and Technology, 430000 Wuhan, China; \\ ccrong@hust.edu.cn (C.R.); xiong_tao@hust.edu.cn (X.T.); zhaoyanghu@hust.edu.cn (Z.H.); \\ xthuang@hust.edu.cn (X.H.), yqzeng@hust.edu.cn (Y.Z.) \\ 2 School of Physics, Huazhong University of Science and Technology, 430000 Wuhan, China; \\ conghuilu@hust.edu.cn \\ * Correspondence: mhliu@hust.edu.cn
}

Received: 1 November 2018; Accepted: 18 December 2018; Published: 21 December 2018

Featured Application: The proposed Wireless Power Transfer (WPT) system with metamaterials using Class-E amplifier can be applied for mid-range high-power energy transfer.

\begin{abstract}
In this paper, a mid-range wireless power transfer (WPT) system based on metamaterials (MMs) has been presented. It has been shown that the MMs are positioned in the WPT system to focalize the electromagnetic field for distance enhancement and efficiency improvement theoretically and experimentally. The MMs were fabricated by using a single layer printed-circuit board (PCB) with the negative magnetic permeability, $\mu_{\mathrm{r}}$. An applicable impedancetuning technology was implemented by changing the operating distance between the drive (load) resonator and the internal resonator, which can achieve the optimal load of the system. In addition, the Class-E RF (radio frequency) power amplifier is firstly proposed as the high frequency excitation source of the WPT system based on the MMs due to its simple design and high efficiency. The proposed technology can achieve efficiency improvements of $4.26 \%$ and $9.13 \%$ at distances of $100 \mathrm{~cm}$ and $200 \mathrm{~cm}$ around the $2.80 \mathrm{MHz}$ WPT system with the MMs, respectively. Specially, it is worth mentioning that the system efficiency is enhanced by $18.58 \%$ at $160 \mathrm{~cm}$. The measured results indicate the WPT system based on the MMs can assure a stable output power of $5 \mathrm{~W}$ at a transfer distance of $200 \mathrm{~cm}$.
\end{abstract}

Keywords: Mid-range wireless power transfer; metamaterials; Class-E RF power amplifier

\section{Introduction}

Wireless power transfer (WPT) via magnetic resonance coupling has extensive application prospects in people's daily lives, such as electrical vehicle charging, home application, portable electronic devices, and robotic applications [1-4]. The transfer characteristics of special WPT systems and promotion of the practicability have become the major hotspots in the WPT research field. In recent studies, the mid-range WPT system mainly concentrates on four methods: (i) Traditional two-coil system, (ii) four-coil system with/without metamaterials (MMs), (iii) WPT system with relay resonators, and (iv) WPT system via a magneto-inductive wave. These four methods have their unique strengths and weaknesses. Additionally, they were analyzed and contrasted specifically in [5]. The principle of wireless power transfer based on magnetically coupled resonance is to take advantage of the resonance coupling of high quality factor coils to realize energy exchange in the near field. The energy 
exchange ratio and transfer loss are determined by the coupling strength and the quality factor of coils. In the mid-range WPT system, the system energy efficiency and transmission distance are important indicators.

In mid-range operation, the transfer distance is usually 50-200 cm [6]. The magnetic coupling coefficient between the two coils decreases sharply when the transfer distance increases. In recent years, a number of research methods have been adopted to analyze the mid-range WPT system. Duong et al. [7] presented an effective method using the "matching condition" technology, which is based on adaptively varying the coupling coefficient between the drive (load) coil and transmission (receiver) coil for the impedance matching to achieve maximum efficiency. Zhang et al. [8] introduced three significant factors in the four-coil structure of resonant WPT and simplified the circuit calculate formula to attain maximum transfer efficiency and output power. Pinuela et al. [9] manifested that the system de-to-load efficiency based on the two-coil inductive mode can be improved greatly by using a Class-E power amplifier, and the system also employed high $\mathrm{Q}$ factor coils operating at about $6 \mathrm{MHz}$. Reference [10-12] employed the physics of the magneto-inductive wave method to explain the behavior of the WPT system with several planar printed resonators in which each capacitive loaded resonator was coupled magnetically to a series of other resonators. The studies in [13-15] reported relay resonators applied between the drive loop and load loop to improve the transmission efficiency and increase the transfer distance operating at a high-frequency situation. The works in [16-22] showed that the negative permeability MMs enhanced the system efficiency and transmission distance due to its abilities of controlling the magnetic field and evanescent wave amplification. However, most research with the MMs has used the scattering parameter (mainly the $S_{21}$ parameter) to describe the transfer efficiency by the network analyzer experimentally, rather than practical system energy efficiency, let alone using the Class-E power amplifier as a high frequency source to drive the whole WPT system with the MMs.

In this paper, we adopt the four-coil WPT system based on magnetically coupled resonance with the MMs in the near field. We firstly extended original research of the four-coil WPT system to elucidate a number of crucial system concepts, including the maximum power efficiency and the optimal load. Then, a single-layer square thin unit cell of loaded spiral resonators was designed. Accordingly, these units were assembled in a $5 \times 5$ slab periodically to achieve a negative magnetic permeability. Furthermore, the tuning scheme was proposed to contribute to the transfer property. In addition, the Class-E power amplifier was employed as the inverter circuit topology to adapt to the highly operating frequency of $2.80 \mathrm{MHz}$. By applying this technology, the system energy efficiency could be improved by $4.26 \%$ and $9.13 \%$ at distances of $100 \mathrm{~cm}$ and $200 \mathrm{~cm}$, respectively. The proposed system is significant as a power supply for electric applications in relatively far distances, such as a mobile phone and tablet PC. In particular, this technology can make the power grid much smarter by supplying high voltage power pole tower on-line monitoring terminal devices.

\section{Proposed WPT System with the MMs}

It is well known that the four-coil WPT system based on magnetic resonance coupling consists of the drive coil, the transmitting coil ( $T x)$, the receiving coil $(R x)$, and the load coil. Normally, the distance between the drive coil (load coil) and the $T x(R x)$ coil is relatively close, so the magnetic coupling is relatively strong. Nevertheless, the $T x$ coil is far from the $R x$ coil and the coupling coefficient is low conversely. Figure 1 presents a diagram of the four-coil WPT system with the MMs. The adjustable DC power source supplies the Class-E amplifier, and the signal generator can emit a high frequency Pulse Width Modulation (PWM) square wave. The Class-E amplifier is designed to amplify the power of a high frequency signal to meet the requirement of transmitting power. The MMs is placed between the $T x$ and $R x$ coil, the position of which is varied constantly and the optimal position of the MMs needs to be analyzed. The load resistance is the link to the load loop. 


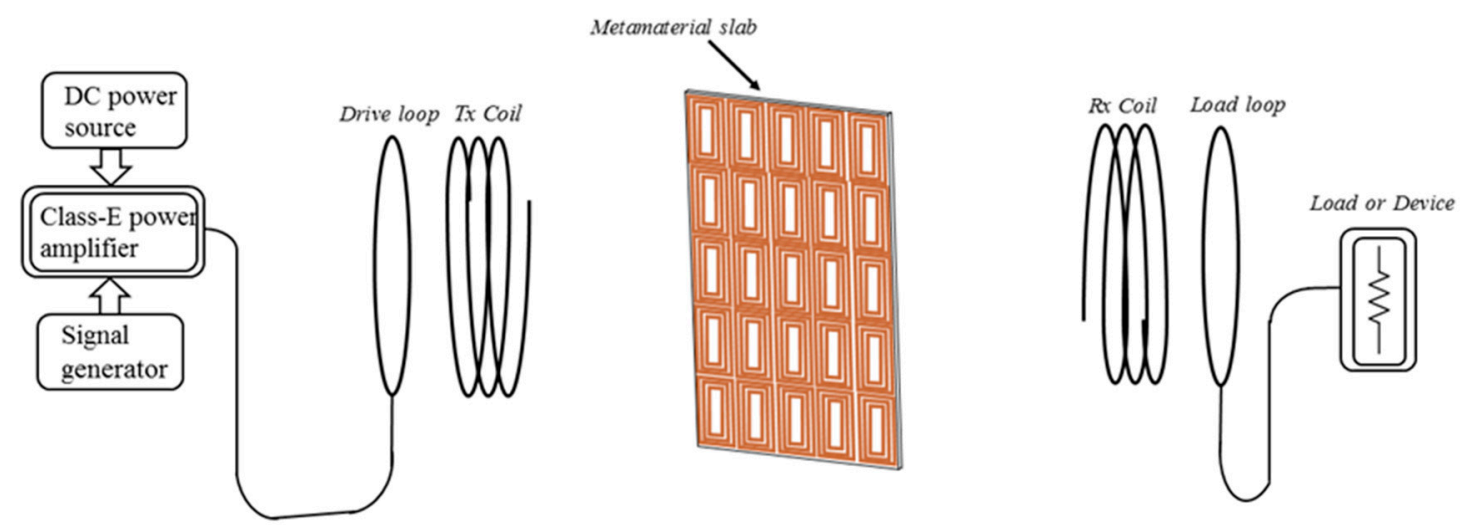

Figure 1. The architecture of the Wireless Power Transfer (WPT) system based on the metamaterials (MMs).

\subsection{Theoretical Analysis of the Four-Coil WPT System}

Figure 2 shows the equivalent circuit diagrams of the four-coil WPT system. All the parameters are considered in the complete model. As a contrast, the equivalent model can be simplified to ignore the less influential quantities. The internal resistance of the power source and the load equivalent resistance are $R_{S}$ and $R_{L}$, respectively. The signal source is denoted by Vs. The distance of the $T x$ coil and $R x$ coil is defined as the transfer distance in the four-coil WPT system. The electrical parameters of each coil are denoted by $R_{i} L_{i} C_{i}(i, j=1,2,3,4) . M_{i j}$ is the mutual inductance of two adjacent coils, and the cross coupling parameters are negligible $\left(M_{13}=M_{24}=M_{14}=0\right)$. Compared with the Rs and $R_{L}$, the coil equivalent resistance of the drive loop and the load loop are so tiny so are neglected. In addition, the four coils have same operating resonance frequency. The simplified circular model is illustrated in Figure 2b.

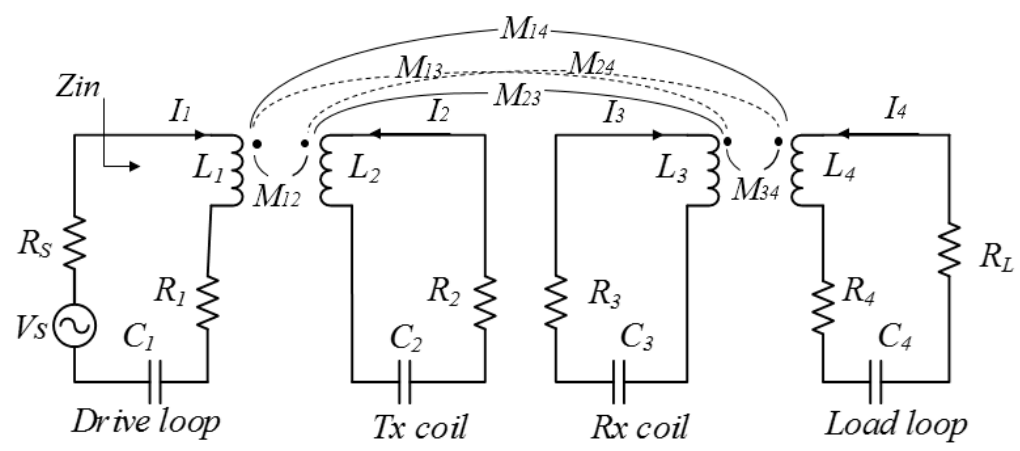

(a)

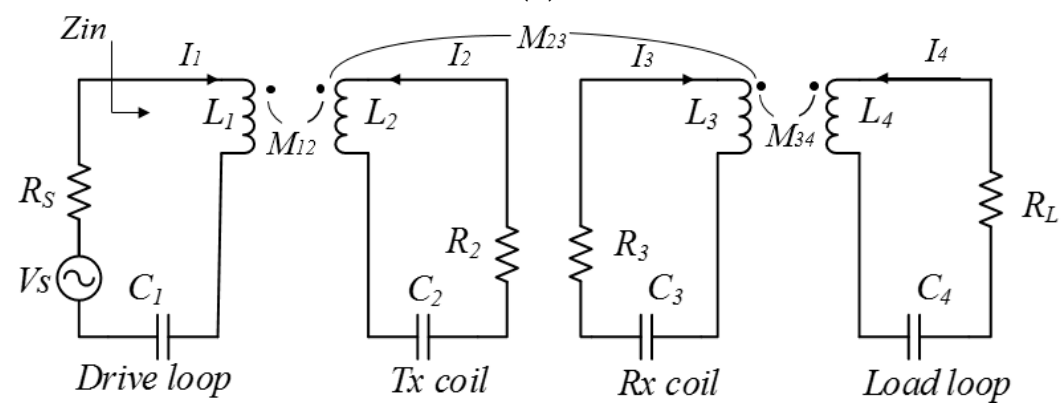

(b)

Figure 2. The equivalent circuit model of the four-coil WPT system. (a) The complete model. (b) The simplified model. 
When the proposed four coils work at same resonant frequency of $2.80 \mathrm{MHz}$, according to the Kirchhoff voltage law (KVL) and the mutual coupling theories, the voltage and current in each coil can be illustrated as follows:

$$
\left\{\begin{array}{l}
V_{s}=R_{s} I_{1}+j \omega M_{12} I_{2} \\
0=R_{2} I_{2}+j \omega M_{12} I_{1}+j \omega M_{23} I_{3} \\
0=R_{3} I_{3}+j \omega M_{23} I_{2}+j \omega M_{34} I_{4} \\
0=R_{L} I_{4}+j \omega M_{34} I_{3}
\end{array}\right.
$$

Similarly, the input impedance, $Z_{i n}$, is a very significant parameter for the design of Class-E, and it can be expressed as:

$$
Z_{\text {in }}=\frac{V_{s}-R_{S} I_{1}}{I_{1}}
$$

Solving (1) and (2), the $Z_{\text {in }}$ can be derived [7], which is:

$$
Z_{\text {in }}=R_{s} \cdot \frac{k_{12}^{2} Q_{1} Q_{2}\left(1+k_{34}^{2} Q_{3} Q_{4}\right)}{1+k_{23}{ }^{2} Q_{2} Q_{3}+k_{34}{ }^{2} Q_{3} Q_{4}}
$$

where $Q_{i}$ is the quality factor of the $i$ th coil. $Q_{1}$ is equal to $Q_{4}$ and $Q_{2}$ is the same as the $Q_{3}$ due to the symmetry of the proposed system. $k_{12}, k_{23}$, and $k_{34}$ are the coupling coefficients of the drive loop and the $T x$ coil, the $T x$ coil and the $R x$ coil, and the load loop and the $R x$ coil, respectively. When the distance between the coils changes, the coupling coefficient varies correspondingly, and eventually the $Z_{\text {in }}$ changes, as shown in the Figure 3. It can be seen that as $k_{12}$ and $k_{34}$ increase, the efficiency also is improved.

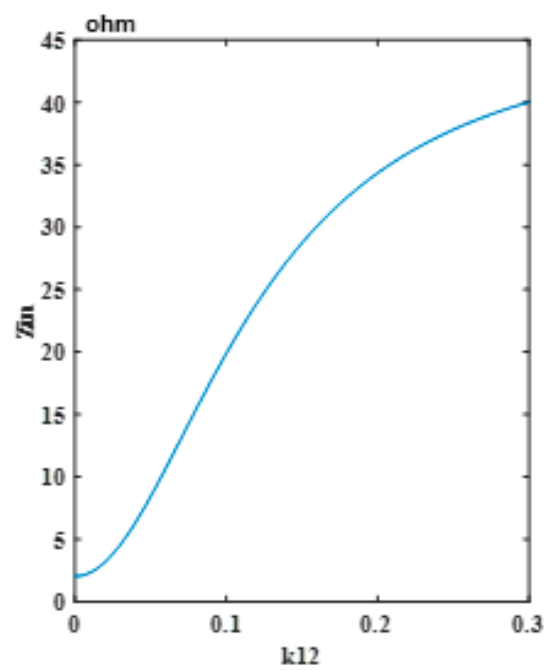

(a)

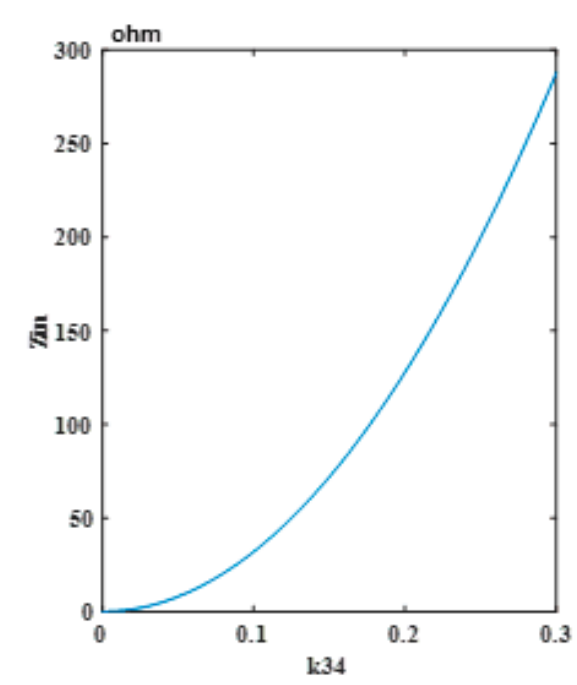

(b)

Figure 3. The relationship (a) between input impedance $Z_{\text {in }}$ and $k_{12}$, and (b) between input impedance $Z_{\text {in }}$ and $k_{34}$.

For a traditional two-coil WPT system, the secondary coil can be reflected as the pure resistance when two coils are in resonance. By adjusting the load resistance value, the system can achieve the maximum efficiency output, namely, there is an optimal load in the system. The optimal load can be expressed as [23]:

$$
R_{L}=\sqrt{1+k^{2} Q_{1} Q_{2}} \cdot R_{2}
$$

in which $k$ is the coupling coefficient, $Q i$ is the quality factor of the $i$ th coil, and $R_{2}$ is the resistance of the secondary coil. Similarly, the four-coil WPT system can be equivalent to the traditional two-coil resonator. The drive loop can be reflected to the Tx coil and the load loop can be reflected in the $R x$ 
coil, as shown in Figure 4 . Rseqt, Vseqt, and $R_{L}$ eqt is the equivalent resistance, voltage supply, and the load resistance, respectively.

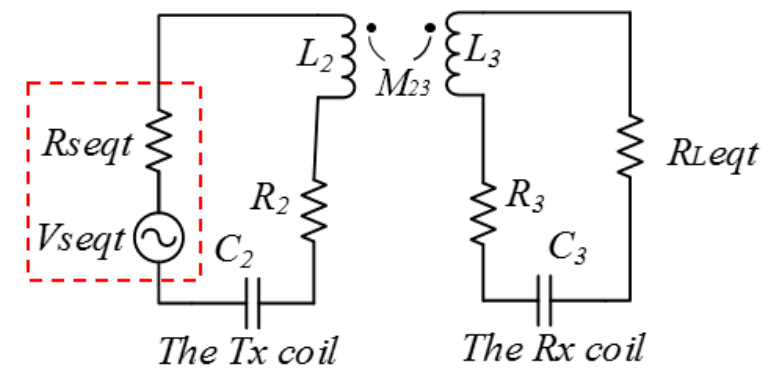

Figure 4. Equivalent simplified circuit diagram of the four-coil WPT system.

According to the two-coil system optimal load analysis, the optimal reflected resistance, $R_{L} e q t$, from the load loop to the $R x$ coil in the four-coil WPT system can be derived as:

$$
R_{\text {Leqt }}=\frac{\left(\omega M_{34}\right)^{2}}{R_{4}+R_{L}}=\sqrt{1+k_{23}{ }^{2} Q_{2} Q_{3}} \cdot R_{3}
$$

From the above formula, to adjust the coupling coefficient between the load loop and the $R x$ coil, namely, to change the distance between the load loop and the $R x$ coil, an optimal load and improvement of the transfer efficiency of the system can be achieved.

In the four-coil WPT system, the system transfer efficiency can be denoted by the power distribution among the equivalent reflected resistances in each coil. The total efficiency is equal to the product of the equivalent resistance ratios. Thus, when four coils are tuning to the resonance frequency, the efficiency is calculated as:

$$
\eta=\frac{\frac{\left(w M_{34}\right)^{2}}{R_{L}}}{\frac{\left(w M_{34}\right)^{2}}{R_{L}}+R_{3}} \frac{\frac{\left(w M_{23}\right)^{2}}{\frac{\left(w M_{34}\right)^{2}}{R_{L}}+R_{3}}}{\frac{\left(w M_{23}\right)^{2}}{\frac{(w 4}{2} M_{3}^{2}}+R_{3}}+R_{2} \frac{\frac{\left(w M_{12}\right)^{2}}{\frac{\left(w M_{23}\right)^{2}}{R_{L}}+R_{2}}}{\frac{\frac{\left(w M_{12}\right)^{2}}{\left(w M_{23}\right)^{2}}+R_{2}}{\frac{\left(w M_{34}\right)^{2}}{R_{L}}+R_{3}}+R_{S}}
$$

The efficiency can be expressed in another form [24]:

$$
\eta=\frac{I_{4}^{2} R_{L}}{\mathrm{~V}_{S} I_{1}}=\frac{\left(k_{12}^{2} Q_{1} Q_{2}\right)\left(k_{23}^{2} Q_{2} Q_{3}\right)\left(k_{34}^{2} Q_{3} Q_{4}\right)}{\left[\left(1+k_{12}^{2} Q_{1} Q_{2}\right)\left(1+k_{34}^{2} Q_{3} Q_{4}\right)+k_{23}^{2} Q_{2} Q_{3}\right]\left[\left(1+k_{23}^{2} Q_{2} Q_{3}+k_{34}^{2} Q_{3} Q_{4}\right)\right]}
$$

Figure 5 shows the system efficiency, $\eta$, as a function of $k_{12}$ and $k_{34}$. It can be seen that $k_{34}$ has a greater effect on the efficiency than $k_{12}$. Generally, when the transfer distance between the $T x$ coil and $R x$ coil is fixed, the maximum efficiency can be achieved by adjusting the distance between the $R x$ coil and the load loop.

In a practical experiment, the distance between the $T x$ coil and the $R x$ coil is fixed at some value ( $k_{23}$ is fixed value), and $k_{12}$ and $k_{34}$ are varied by adjusting the distance between relevant coils to change the $Z_{\text {in }}$ and system efficiency in the meantime. From the optimal load analysis of the four-coil WPT system, the $k_{34}$ is an important factor to the optimal load. In addition, the $k_{34}$ has a greater influence than the $k_{12}$ on the $Z_{\text {in }}$ and system efficiency. In general, we fixed the distance between the $T x$ coil and the $R x$ coil and varied the distance between the load loop and the $R x$ coil to achieve the optimal load, and then changed the distance between the drive loop and the $T x$ coil to attain the needed $Z_{\text {in }}$ of the Class-E amplifier. 


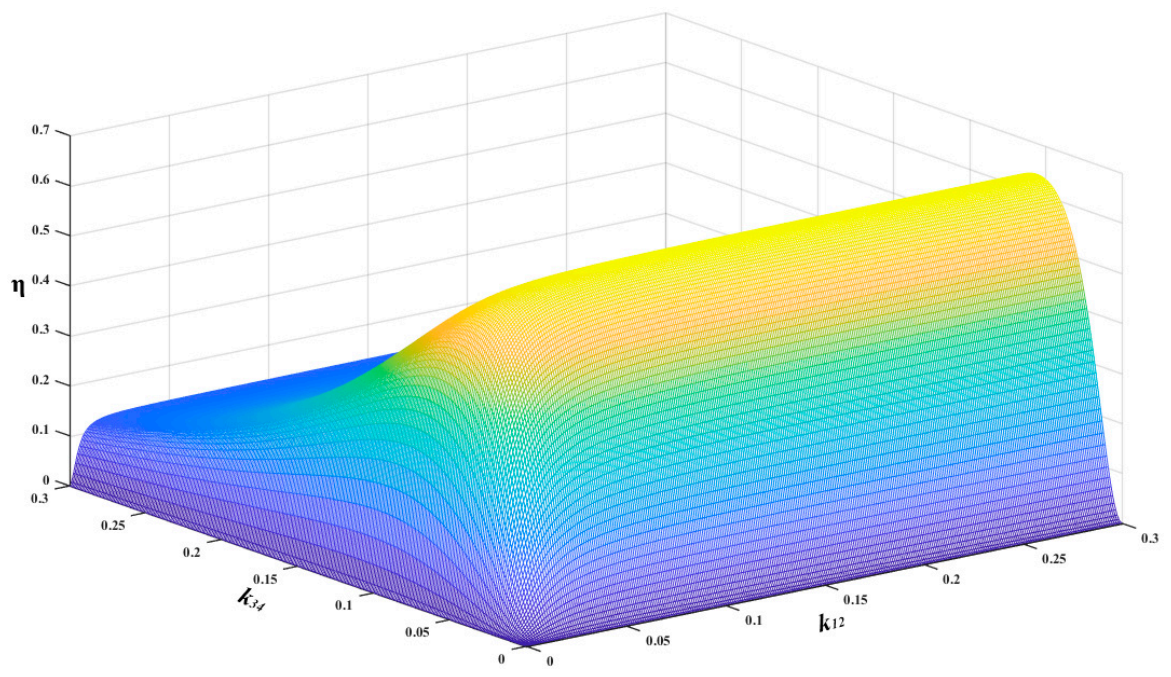

Figure 5. The efficiency as a function of the $k_{12}$ and the $k_{23}$.

\subsection{Analysis and Design of the MMs}

It is generally known that the MMs with negative permeability have the supernatural ability that changes the direction of the magnetic propagation and contributes to distance enhancement and efficiency improvement. Reference [20-22] used the numerical models and analytical theory to explain the principle of MM's focusing property. According to Maxwell equations:

$$
\left\{\begin{array}{l}
k \cdot E=0 \\
k \cdot H=0 \\
k \times E=\mu \omega H \\
k \times H=-\varepsilon \omega E
\end{array}\right.
$$

where $w$ is the plane electromagnetic wave frequency, $k$ is wave vector, $\mu$ is the dielectric permeability, $\varepsilon$ is the dielectric constant, $E$ is the electric field intensity, and $H$ is the magnetic field intensity. According to the formula, $n^{2}=\mu_{r} \varepsilon_{r}$, when $k, E$, and $H$ form the left hand system, and the refractive index, $n$, is negative. When the electromagnetic wave enters the surface of the medium, according to Fresnel's law:

$$
\frac{\tan \theta_{1}}{\tan \theta_{2}}=\frac{\mu_{r 1}}{\mu_{r 2}}
$$

$\theta_{1}$ and $\theta_{2}$ are the angle of incidence and refraction. As shown in Figure 6, the refraction occurs when electromagnetic waves pass through two dielectric interfaces with opposite chirality. Figure $6 \mathrm{c}$ manifests that the reflected wave and the incident wave are in the same direction when the relative permeability is negative. If the magnetic field can be restrained effectively and controlled artificially to solve the problem of rapid divergence and attenuation of the magnetic field, the transmission distance and efficiency of the magnetic resonance wireless transfer system can be improved effectively [18].

To achieve the desired MMs slab, the HFSS (High Frequency Simulator Structure) software was adopted to simulate the MM structure. The S-parameter can be obtained and finally converted into the relationship between the relative permeability and frequency [25-27]. To make the MM unit cell elastic and compact, the proposed MM unit cell was designed to be square with a length of $120 \mathrm{~mm}$ and a series capacitance of $2.27 \mathrm{nF}$ was welded on both ends of the copper strip to tune the resonant frequency of the MM unit cell. The structure diagram of the MM unit cell is shown in Figure 7a. The substrate was made of FR- 4 whose relative dielectric constant was $\varepsilon r=4.4$ and the loss tangent was $\tan \delta=0.025$ and its thickness was $2 \mathrm{~mm}$. The extracted relative permeability curve depending on the frequency of the MM unit cell is illustrated in Figure $7 \mathrm{~b}$. The structure parameters of the MM unit cell are listed in Table 1. 


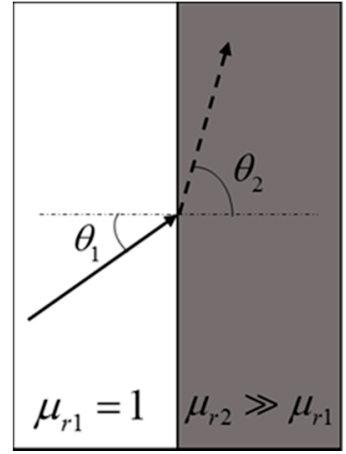

(a)

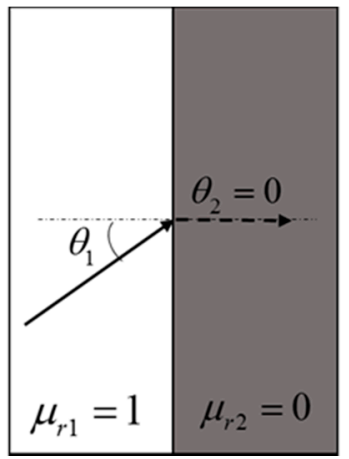

(b)

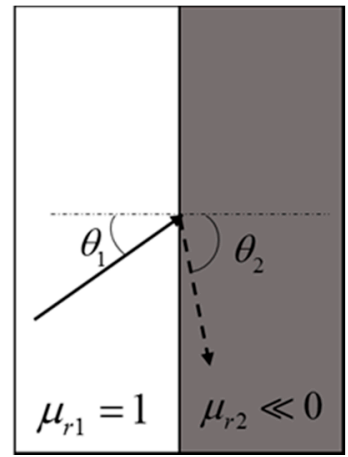

(c)

Figure 6. Reflection principle of electromagnetic waves. (a) High positive $\mu_{\mathrm{r} 2}$. (b) Zero $\mu_{\mathrm{r} 2}$. (c) High negative $\mu_{\mathrm{r} 2}$.

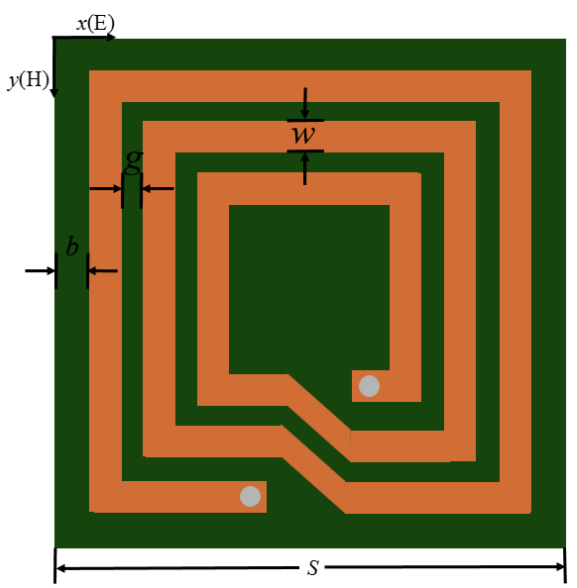

(a)

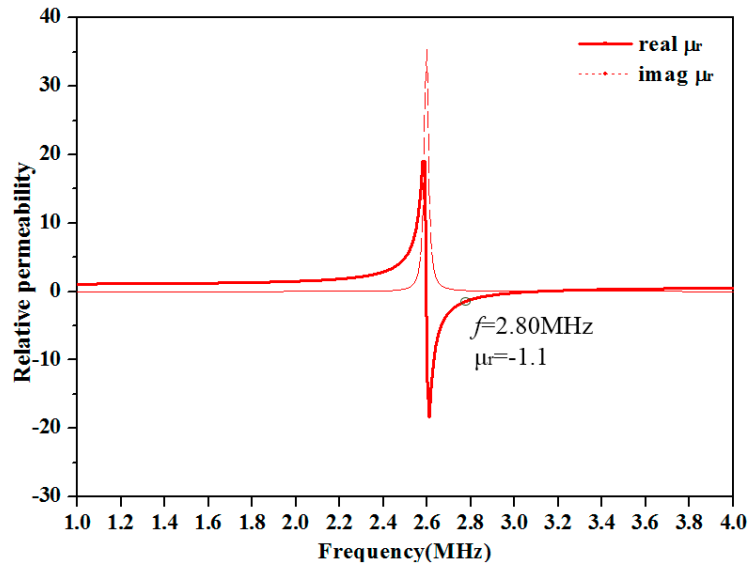

(b)

Figure 7. (a) The structure of the MM unit cell. (b) The relative permeability versus the frequency of the MM unit cell.

Table 1. The structure parameters of the MM unit cell.

\begin{tabular}{ccc}
\hline Symbol & Parameter & Value \\
\hline $\mathrm{S}$ & Length of side $(\mathrm{mm})$ & 120 \\
$\mathrm{~g}$ & Interval $(\mathrm{mm})$ & 3 \\
$\mathrm{~W}$ & Width $(\mathrm{mm})$ & 4 \\
$\mathrm{~N}$ & Turn & 3 \\
$\mathrm{~T}$ & Thickness of substrate & 2 \\
\hline
\end{tabular}

To optimize the MM unit cell design, a variety of parameter structures were compared for resonance frequency control, as shown in Figure 8. When the width, the interval and the turn of the copper strip, and the thickness of the substrate varied, the resonance frequency of the MM unit cell changed correspondingly. It can be seen that the width and interval had no obvious influence on the resonance frequency, whereas the change of the turn made the resonance frequency deviate largely. The main reason was that the increase of the turn number made the inductance increase more distinct. In addition, the thickness of the substrate had less impact for the resonance frequency. In this paper, the MM unit cell adopting the above structure parameters was a comprehensive consideration of size, flexibility, and firmness in fabrication. To accommodate the coil size, the MMs slab consisted of a $5 \times 5$ unit cell, and the diameter of the $T x(R x)$ coil was $500 \mathrm{~mm}$. 


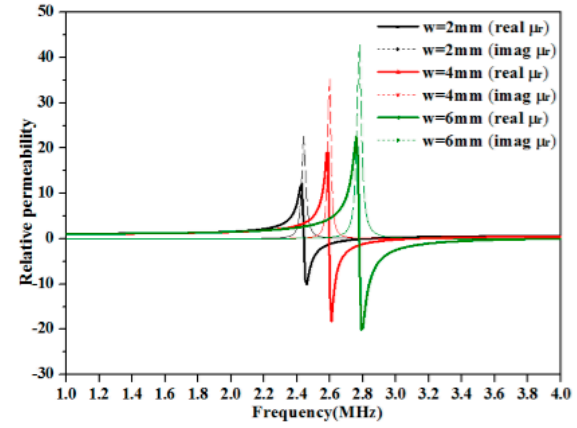

(a)

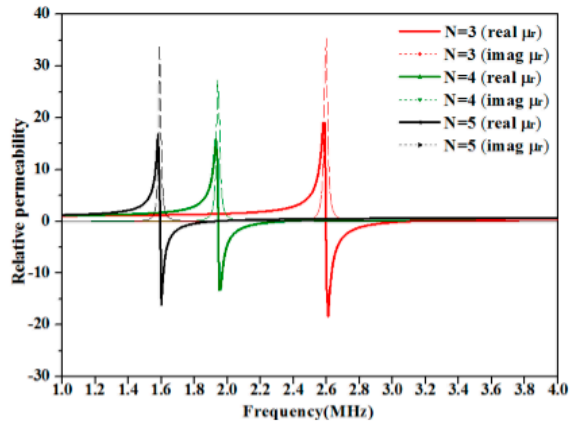

(c)

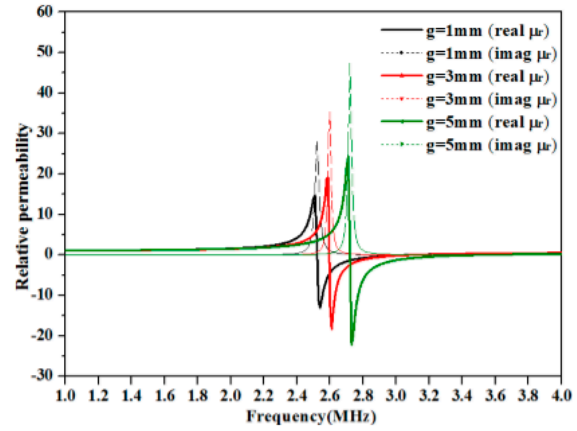

(b)

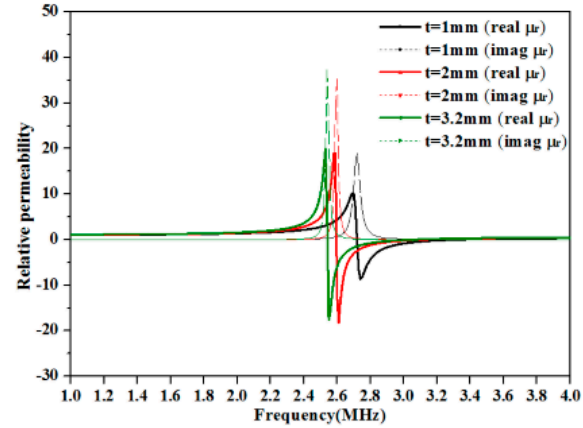

(d)

Figure 8. The relative permeability as a function of the resonance frequency of the MM unit cell by varying: (a) The width of the copper strip; (b) the interval between the copper strips; (c) the turn number of the copper strip; and (d) the thickness of the substrate.

\section{Fabrication of Class-E Amplifier and Experiment Verification}

To verify the previous power transfer efficiency analysis and the effect of MMs, a high-frequency Class-E power drive was designed. The Class-E amplifier has a simple topology and operating frequency up to the $\mathrm{MHz}$ level. It is suitable to be used as a high frequency excitation source for the WPT system with the MM. The Class-E amplifier consists of the DC voltage source $V_{I}$, RF choke $L_{f}$, shunt capacitance, $C_{1}$, Metal-Oxide-Semiconductor Field Effect Transistor (MOSFET), $S$, resonant capacitance, $C$, and inductor, $L$. It can reach the soft switching state with the appropriate parameter of the electrical component [28-30]. Furthermore, the RF Class-E topology needs to be chosen to meet the four-coil system property. Figure 9 shows the circuit sketch of the WPT system with the Class-E amplifier. We set the equivalent output resistance of the Class-E to $10 \Omega$, namely, the Zin needed to be adjusted to $10 \Omega$ by varying the distance between coils. The operating frequency of system was $2.80 \mathrm{MHz}$. The quality factor was set as 8 . The rest of the parameters were calculated by expression (10). Table 2 lists the parameters of the Class-E amplifier.

$$
\left\{\begin{array}{l}
L_{f}=2\left(\frac{\pi^{2}}{4}+1\right) \frac{R}{f} \approx \frac{7 R}{f} \\
C_{1}=\frac{8}{\pi\left(\pi^{2}+4\right) w R} \\
C=\frac{1}{w R\left[Q-\frac{\pi\left(\pi^{2}-4\right)}{16}\right]} \\
L=\frac{Q R}{w}
\end{array}\right.
$$




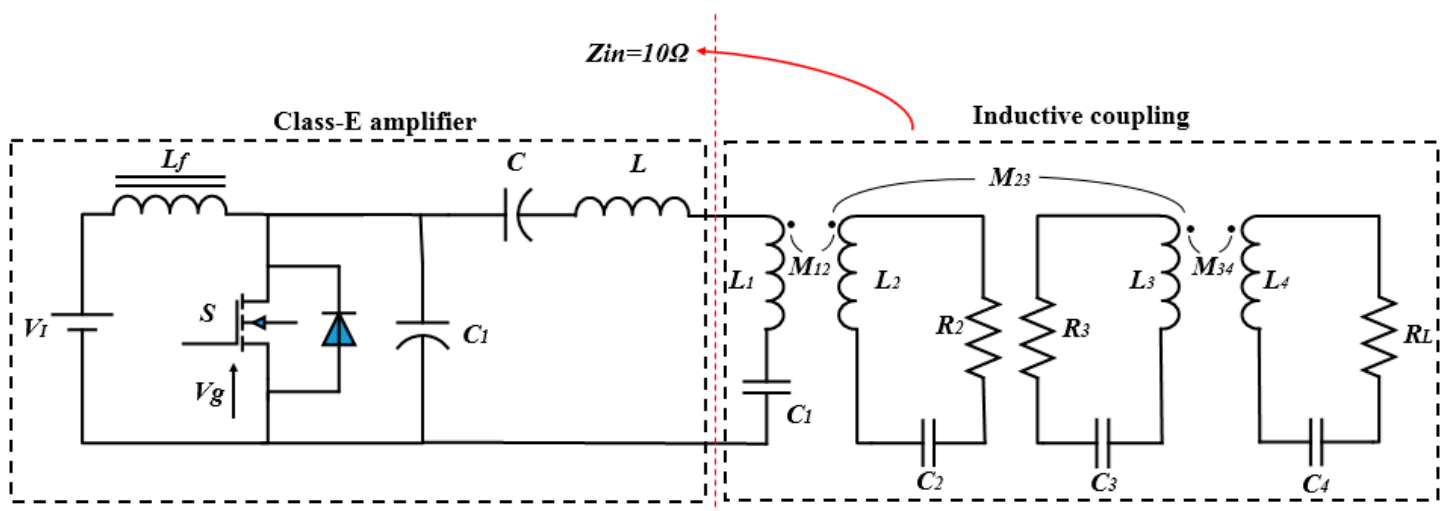

Figure 9. The equivalent circuit diagram with the Class-E amplifier.

Table 2. Parameters of the proposed Class-E amplifier.

\begin{tabular}{ccc}
\hline Meaning & Symbol & Value \\
\hline Resonant frequency & $\mathrm{f}$ & $2.80 \mathrm{MHz}$ \\
Shunt capacitance & $\mathrm{C}_{0}$ & $1.04 \mathrm{nF}$ \\
Resonant inductance & $\mathrm{L}$ & $4.54 \mathrm{uH}$ \\
Resonant capacitance & $\mathrm{C}$ & $0.83 \mathrm{nF}$ \\
Quality factor & $\mathrm{Q}$ & 8 \\
Choke inductance & $\mathrm{L}_{0}$ & $31.56 \mathrm{uH}$ \\
\hline
\end{tabular}

A few megahertz switching frequency and hundreds of watts can be achieved by the using of silicon carbide devices (C2M008012D), and its conduction resistance and parasitic capacitance are very tiny. The driver chip (IXDD614) was employed in this circuit template, as shown in Figure 10a. Figure $10 \mathrm{~b}$ shows that voltage waveform of the test load resistance, the waveform was a sinusoidal wave approximately and its working frequency was $2.80 \mathrm{MHz}$. The duty ratio of the signal was less than $50 \%$, ensuring that the amplifier operated in a soft-switched state.

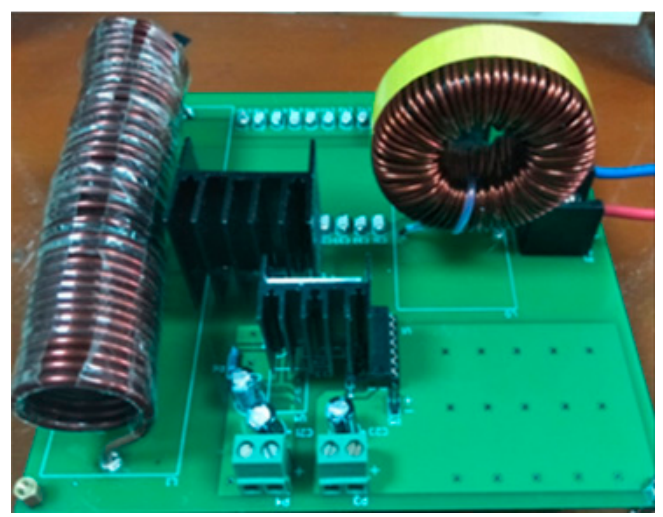

(a)

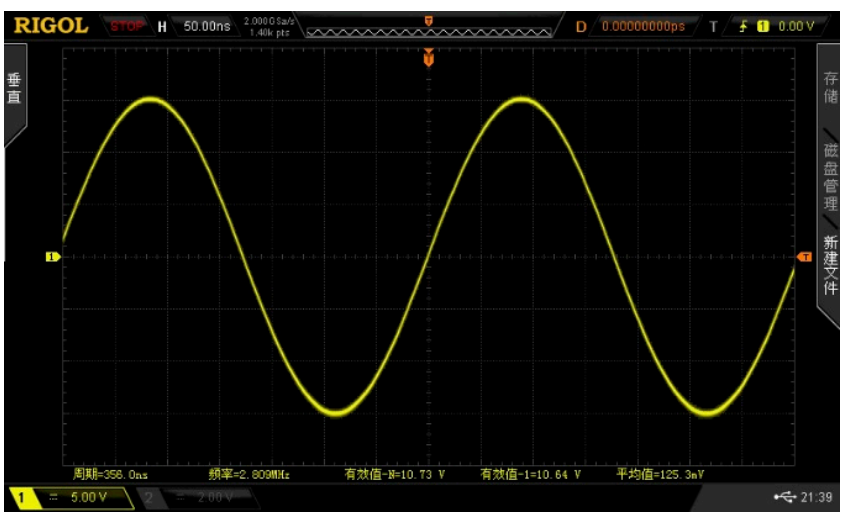

(b)

Figure 10. (a) The physical circuit board of each component of the Class-E amplifier. (b) The voltage waveform of the test load resistance.

To verify the proposed the method, a four-coil WPT system with the MMs was built, as shown in Figure 11. The radius of the Tx coil was set to $25 \mathrm{~cm}$, a turn of 10, and a pitch of $4 \mathrm{~cm}$, with the radius of the copper tube as $4 \mathrm{~mm}$, the same as the $\mathrm{Rx}$ coil. Additionally, both the drive loop and the load loop were the single turn circular coil with a diameter of $40 \mathrm{~cm}$. The additional capacitor was 
welded to the four coils to achieve a resonant frequency of $2.80 \mathrm{MHz}$. The parameters of the four-coil WPT system is shown in Table 3. For simplification, the skin effect and proximity effect were ignored. The unit cells were assembled periodically as a $5 \times 5 \mathrm{MMs}$ slab. The MMs slab was located between the $T x$ coil and the $R x$ coil. All the modules, including the drive loop, the Tx coil, the MMs slab, the $R x$ coil, and the load loop, were placed coaxially. The DC power source supplied energy for the Class-E amplifier, the signal generator emitted the high frequency PWM electrical level, and the oscilloscope measures the waveform of the output resistance. The MMs was located between the $R x$ coil and the $T x$ coil and the position of the MMs changed along with the distance between the $R x$ coil and the $T x$ coil accordingly.

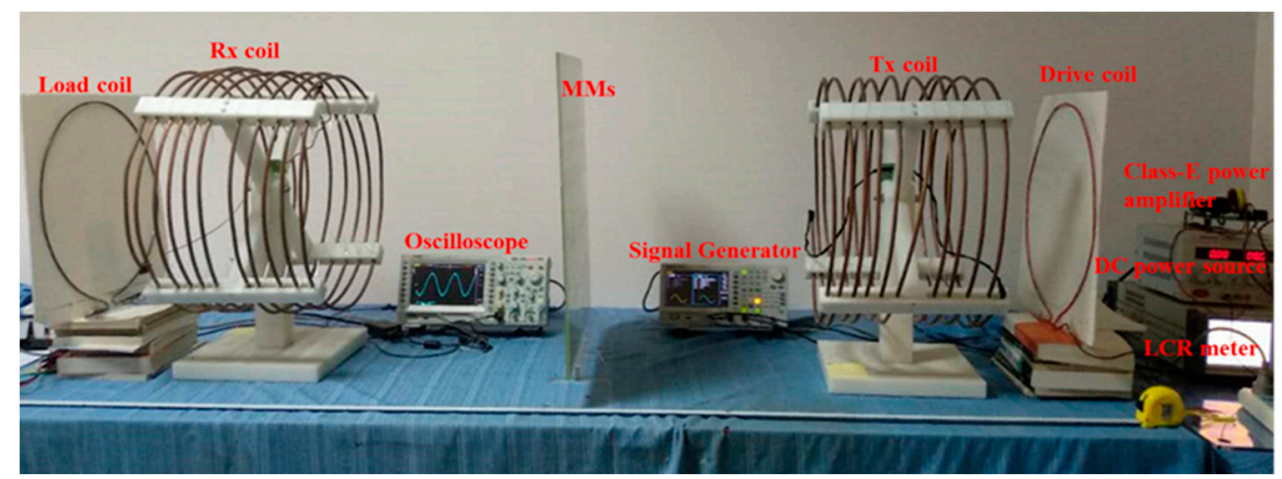

Figure 11. Experiment setup of the WPT system with the MM slab.

Table 3. The electrical parameters of the four coils.

\begin{tabular}{ccccccc}
\hline & $\begin{array}{c}\text { Inductance } \\
(\mathbf{u H})\end{array}$ & $\begin{array}{c}\text { Resistance } \\
(\boldsymbol{\Omega})\end{array}$ & $\begin{array}{c}\text { Parasitic } \\
\text { Capacitor }(\mathbf{p F})\end{array}$ & $\begin{array}{c}\mathbf{Q} \text { (Unload) } \\
\mathbf{0} \mathbf{2 . 8 0} \mathbf{~ M H z}\end{array}$ & $\begin{array}{c}\text { Additional } \\
\text { Capacitor }(\mathbf{p F})\end{array}$ & $\begin{array}{c}\text { Resonant } \\
\text { fre. (MHz) }\end{array}$ \\
\hline Drive coil & 1.76 & 0.1 & - & 110 & $1.835 \times 10^{3}$ & 2.81 \\
\hline$T x$ coil & 49.6 & 1.64 & 10.9 & 532.1 & 50 & 2.80 \\
$R x$ coil & 49.1 & 1.63 & 10.5 & 531.8 & 50 & 2.81 \\
Load coil & 1.75 & 0.1 & - & 109 & $1.835 \times 10^{3}$ & 2.80 \\
\hline
\end{tabular}

Firstly, we changed the distance between the load loop and the $R x$ coil to achieve the optimal load, and then changed the distance between the drive loop and the Tx coil to attain the needed Zin of the Class-E amplifier. The LCR meter was to make sure that the output resistance, Zin, was equal to about $10 \Omega$ by varying the distance between the two coils.

Figure 12 shows the optimal load by changing the distance between the load loop and the $R x$ coil in the four-coil WPT system without MMs. $d_{t l}$ denotes the distance between the load loop and the $R x$ coil. $d_{t r}$ represents the distance between the $T x$ coil and the $R x$ coil. When the $d_{t r}$ was fixed at a certain value, the $d_{t r}$ was varied to achieve the maximum efficiency. It can be seen that as the system transfer distance, $d_{t r}$, increased, the optimal distance of $d_{t l}$ increased. Changes of the coil distances resulted in variations of the coupling coefficient. This experimental phenomenon is in good agreement with the previous analysis.

Table 4 lists the measured power results of the WPT system with the MMs. The magnitudes of the input current is $I_{1}$, and the input and output voltages are $V_{1}$ and $V_{2}$, respectively. $V_{1}$ and $I_{1}$ were obtained from the DC power source. $V_{2}$ was measured by an oscilloscope. The load resistance, $R_{L}$, was $10 \Omega$, and the output power $P_{L}$ was calculated as the radio of the square of the output voltage, $V_{2}$, to the load resistance, $R_{L}$. The system power efficiency was derived by the expression, $\eta=P_{L} / V_{1} I_{1}$. This table lists a set of measured data and manifests that the system output power can reach $5 \mathrm{w}$ approximately of $20.11 \%$ efficiency when the transfer distance was $200 \mathrm{~cm}$. 


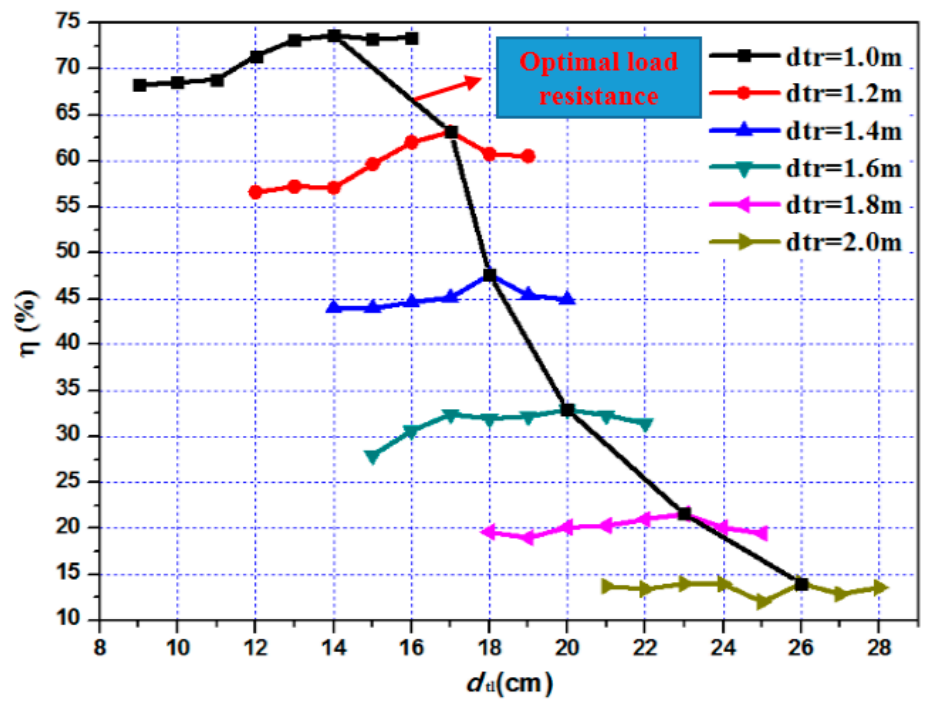

Figure 12. The experiment of the optimal load analysis.

Table 4. Measured power results of the WPT system with the MMs.

\begin{tabular}{cccccc}
\hline & $\mathbf{V}_{\mathbf{1}}(\mathbf{V})$ & $\mathbf{V}_{\mathbf{2}} \mathbf{( V )}$ & $\mathbf{I}_{\mathbf{1}} \mathbf{( A )}$ & $\boldsymbol{\eta} \mathbf{( \% )}$ & $\mathbf{P}_{\mathbf{L}} \mathbf{( W )}$ \\
\hline $100 \mathrm{~cm}$ & 10.5 & 7.14 & 0.62 & 77.90 & 5.10 \\
$120 \mathrm{~cm}$ & 11.4 & 7.16 & 0.61 & 73.64 & 5.12 \\
$140 \mathrm{~cm}$ & 14.3 & 7.16 & 0.62 & 57.03 & 5.13 \\
$160 \mathrm{~cm}$ & 15.1 & 7.14 & 0.71 & 50.92 & 5.10 \\
$180 \mathrm{~cm}$ & 15.73 & 7.16 & 1.05 & 30.87 & 5.13 \\
$200 \mathrm{~cm}$ & 19.25 & 7.15 & 1.31 & 20.11 & 5.12 \\
\hline
\end{tabular}

Figure 13 shows the measured results for the optimal loading position of the MMs in the WPT system. $d_{t r}$ represents the distance between the $T x$ coil and the $R x$ coil and $d_{t m}$ denotes the distance between the $T x$ coil and the MMs. The distance of the $T x$ coil and the $R x$ coil was fixed to a certain value, varying from $1 \mathrm{~m}$ to $2 \mathrm{~m}$, as labeled in the upper right area of the figure. The MM slab was moved $10 \mathrm{~cm}$ apart between the $T x$ coil and the $R x$ coil at the certain $d_{t r}$, as shown in the x-coordinate of the figure. It can be seen that the transfer efficiency decreased when the $d_{t r}$ increased. Furthermore, the transfer efficiency reached the maximum when the MMs slab was placed in the middle of the two coils approximately and the MM's optimum loading position increased with the enhancement of the transmission distance generally.

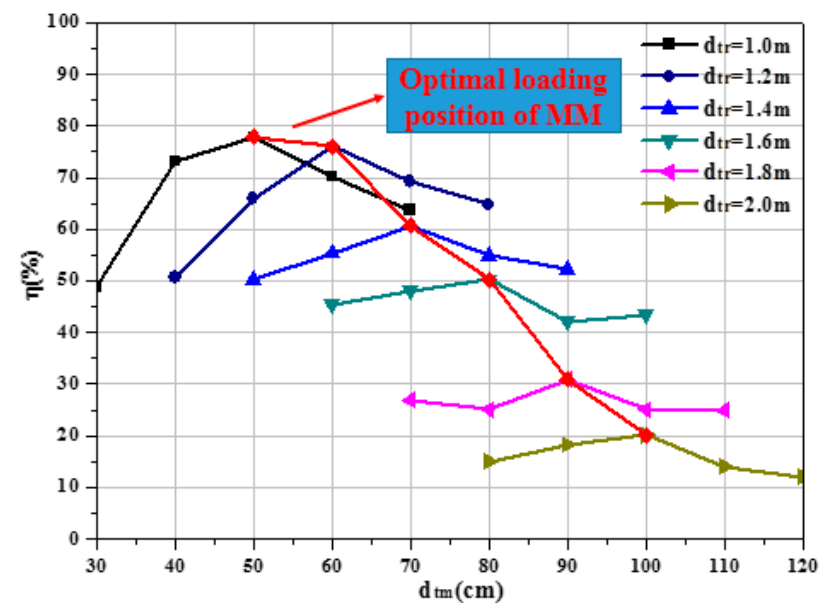

Figure 13. Effect of the MMs' loading position on the system transmission efficiency. 
Figure 14 compares the measured transfer efficiency of the WPT system with/without the MMs. Obviously, the MMs contributed to the efficiency improvement for the mid-range WPT system. The strength of coupling between the $T x$ coil and the $R x$ coil was large when the two coils were closer together, so the MMs have little effect on the efficiency. However, the MMs was very useful for efficiency enhancement as the transfer distance became larger. The transfer efficiency improved by $3.26 \%$ when the $R x$ coil and $T x$ coil were $100 \mathrm{~cm}$ apart. On the contrary, the MMs enabled the WPT system to remain highly efficient when the two coils were far apart from each other. The transfer efficiency was enhanced by $9.13 \%$ when the transfer distance was $200 \mathrm{~cm}$ apart. In particular, the transfer efficiency was maximized up to $18.58 \%$ at $160 \mathrm{~cm}$.

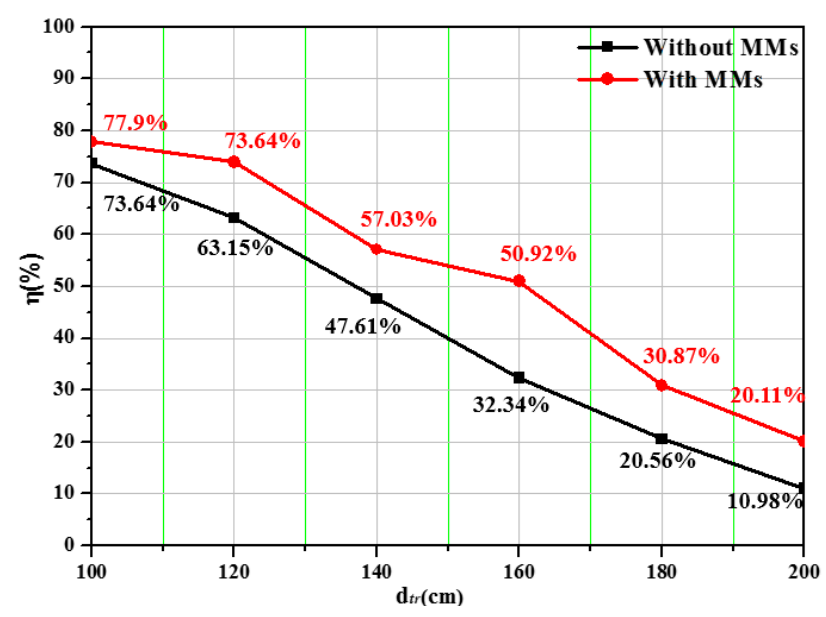

Figure 14. Comparison of transfer efficiency before and after the MMs loading.

\section{Discussion}

This paper discusses the WPT system with the MMs using a Class-E RF power amplifier. It elaborates that the system transfer efficiency remains high in relatively long distances. Research of the WPT system and the MMs have been analyzed separately in recent years. Most studies have explained that the MMs are applied in the WPT system based on the physical mechanisms and simulated analysis, such as the microwave theory and transmission line theory. To explore an effective method to combine the WPT system with the MM is the focus of future research, such as the equivalent circuit model. This method can help to analyze the inner relation between the WPT system and the MMs.

\section{Conclusions}

The mid-range WPT system based on the metamaterials using the Class-E amplifier was analyzed. The principle revealed that the MMs helped with efficiency improvement and distance enhancement in the four-coil WPT system. Furthermore, the Class-E power amplifier applied in the WPT system with the MMs was firstly proposed. The experiment setup exhibited the transfer efficiency in the WPT system with/without the MMs and displayed the MMs' focusing property. In conclusion, the proposed method can be practical for the mid-range WPT system, especially for household electric application and on-line monitoring of terminal devices on the power pole tower.

Author Contributions: C.R. and M.L. conceived and designed the study; C.R. and X.T. gave the theoretical and data analysis; X.H. and C.L. gave the design of metamaterials; Z.H. and Y.Z. revised the whole manuscript; M.L. gave the financial support during the research; C.R. wrote the paper.

Funding: This research was supported by the National Key R\&D Program of China (Grant No. 2018YFB0106300), Scientific Projects of State Grid Corporation of China under Grant No. 0231132705, Huazhong University of Science and Technology. 
Acknowledgments: This work was supported by the National Key R\&D Program of China (Grant No. 2018YFB0106300), Scientific Projects of State Grid Corporation of China under Grant No. 0231132705, Huazhong University of Science and Technology.

Conflicts of Interest: The authors declare no conflict of interest.

\section{References}

1. Zhu, Q.; Wang, L.; Guo, Y.; Liao, C.; Li, F. Applying LCC Compensation Network to DynamicWireless EV Charging System. IEEE Trans. Ind. Electron. 2016, 63, 6557-6567. [CrossRef]

2. Kim, S.M.; Moon, J.K.; Cho, I.K. 120W Wireless Power Transfer System for the Wireless Seat in Automobile. In Proceedings of the 2017 Progress in Electromagnetics Research Symposium, Singapore, 19-22 November 2017; pp. 2635-2637.

3. Roshan, Y.M.; Park, E.J. Design approach for a wireless power transfer system for wristband wearable devices. IET Power Electron. 2017, 10, 931-937. [CrossRef]

4. Basar, M.R.; Ahmad, M.Y.; Cho, J.; Ibrahim, F. Stable and High-Efficiency Wireless Power Transfer System for Robotic Capsule Using a Modified Helmholtz Coil. IEEE Trans. Ind. Electron. 2017, 64, 1113-1122. [CrossRef]

5. Hui, S.Y.R.; Zhong, W.; Lee, C.K. A critical review of recent progress in mid-range wireless power transfer. IEEE Trans. Power Electron. 2014, 26, 4500-4511. [CrossRef]

6. Ren, J.; Hu, P.; Yang, D.; Liu, D. Tuning of mid-range wireless power transfer system based on delay-iteration method. IET Power Electron. 2015, 9, 1563-1570. [CrossRef]

7. Duong, T.P.; Lee, J.W. Experimental Results of High-Efficiency Resonant Coupling Wireless Power Transfer Using a Variable Coupling Method. IEEE Microw. Wirel. Comp. Lett. 2011, 21, 442-444. [CrossRef]

8. Zhang, Y.; Zhao, Z.; Lu, T. Quantitative Analysis of System Efficiency and Output Power of Four-Coil Resonant Wireless Power Transfer. IEEE J. Emerg. Sel. Top. Power Electron. 2015, 3, 184-190. [CrossRef]

9. Pinuela, M.; Yates, D.; Lucyszyn, S.; Mitcheson, P.D. Maximising DC to load efficiency for inductive power transfer. IEEE Trans. Power Electron. 2013, 28, 2437-2447. [CrossRef]

10. Shamonina, E.; Kalinin, V.A.; Ringhofer, K.H.; Solymar, L. Magnetoinductive waves in one, two, and three dimensions. J. Appl. Phys. 2002, 92, 6252-6261. [CrossRef]

11. Stevens, C.J.; Chan, C.W.T.; Stamatic, K.; Edwards, D.J. Magnetic Metamaterials as 1-D Data TransferChannels: An Application for Magneto-Inductive Waves. IEEE Trans. Microw. Theory Tech. 2010, 58, 1248-1256. [CrossRef]

12. Stevens, C.J. Magnetoinductive Waves and Wireless Power Transfer. IEEE Trans. Power. Electron. 2015, 30, 6182-6190. [CrossRef]

13. Zhang, F.; Hackworth, S.A.; Fu, W.; Li, C.; Mao, Z.; Sun, M. Relay effect of wireless power transfer using strongly coupled magnetic resonances. IEEE Trans. Magn. 2011, 47, 1478-1481. [CrossRef]

14. Kiani, M.; Jow, U.M.; Ghovanloo, M. Design and optimization of a 3-coil inductive link for efficient wireless power transmission. IEEE Trans. Biomed. Circuits Syst. 2011, 5, 579-591. [CrossRef] [PubMed]

15. Zhang, X.; Ho, S.L.; Fu, W.N. Quantitative design and analysis of relay resonators in wireless power transfer system. IEEE Trans. Magn. 2012, 48, 4026-4029. [CrossRef]

16. Li, W.; Wang, P.; Yao, C.; Zhang, Y.; Tang, H. Experimental investigation of 1D, 2D, and 3D metamaterials for efficiency enhancement in a $6.78 \mathrm{MHz}$ wireless power transfer system. In Proceedings of the 2016 IEEE Wireless Power Transfer Conference (WPTC), Aveiro, Portugal, 5-6 May 2016; pp. 1-4.

17. Wang, B.; Yerazunis, W.; Teo, K.H. Wireless Power Transfer: Metamaterials and Array of Coupled Resonators. Proc. IEEE 2013, 101, 1359-1368. [CrossRef]

18. Cho, Y.; Kim, J.J.; Kim, D.H.; Lee, S.; Kim, H. Thin PCB-Type Metamaterials for Improved Efficiency and Reduced EMF Leakage in Wireless Power Transfer Systems. IEEE Trans. Microw. Theory Tech. 2016, 64, 353-364. [CrossRef]

19. Ranaweera, A.L.A.K.; Duong, T.P.; Lee, J.W. Experimental investigation of compact metamaterial for high efficiency mid-range wireless power transfer application. J. Appl. Phys. 2014, 116, 83-86. [CrossRef]

20. Boyvat, M.; Hafner, C.V. Molding the Flow of Magnetic Field with Metamaterials: Magnetic Field Shielding. Prog. Electromagn. Res. 2012, 126, 303-316. [CrossRef]

21. Ranaweera, A.L.A.K.; Arriola, C.; Lee, J.W. Anisotropic metamaterial for efficiency enhancement of mid-range wireless power transfer under coil misalignment. J. Phys. D Appl. Phys. 2015, 48, 455104. [CrossRef] 
22. Huang, D.; Urzhumov, Y.; Smith, D.R.; Teo, K.H.; Zhang, J. Magnetic superlens-enhanced inductive coupling for wireless power transfer. J. Appl. Phys. 2012, 111, 064902. [CrossRef]

23. Liu, S.; Chen, L.; Zhou, Y.; Cui, T.J. A general theory to analyse and design wireless power transfer based on impedance matching. Int. J. Electron. 2014, 101, 1375-1404. [CrossRef]

24. Ram Rakhyani, A.K.; Mirabbasi, S.; Chiao, M. Design and Optimization of Resonance-Based Efficient Wireless Power Delivery Systems for Biomedical Implants. IEEE Trans. Biomed. Circuits Syst. 2011, 5, 48-63. [CrossRef]

25. Smith, D.; Schultz, S.; Markos, P.; Soukoulis, C. Determination of effective permittivity and permeability of metamaterials from reflection and transmission coefficients. Phys. Rev. B, Condens. Matter 2002, 65, 195104. [CrossRef]

26. Smith, D.; Vier, D.; Koschny, T.; Soukoulis, C. Electromagnetic parameter retrieval from inhomogeneous metamaterials. Phys. Rev. E Stat. Phys. Plasmas Fluids Relat. Interdiscip. Top. 2005, 71, 036617. [CrossRef] [PubMed]

27. Cheng, Y.Z.; Jin, J.; Li, W.L.; Chen, J.F.; Wang, B.; Gong, R.Z. Indefinite-permeability metamaterial lens with finite size for miniaturized wireless power transfer system. AEU Int. J. Electron. Commun. 2016, 70, 1282-1287. [CrossRef]

28. Carrubba, V.; Maroldt, S.; Musser, M.; Ture, E.; Dammann, M.; van Raay, F.; Quay, R.; Brückner, P.; Ambacher, O. High-Efficiency, High-Temperature Continuous Class-E Sub-Waveform Solution AlGaN/GaN Power Amplifier. IEEE Microw. Wirel. Compon. Lett. 2015, 25, 526-528. [CrossRef]

29. Oh, H.S.; Song, T.; Yoon, E.; Kim, C.K. A power-efficient injection-locked class-E power amplifier for wireless sensor network. IEEE Microw. Wirel. Compon. Lett. 2006, 16, 173-175.

30. Takahashi, E.; Ishikawa, T.; Kashimura, K.; Adachi, N. High-Efficiency Four-Stage Class-E Doherty Amplifier for W-CDMA Base Stations. In Proceedings of the 2008 38th European Microwave Conference, Amsterdam, The Netherlands, 27-31 October 2008; pp. 234-237.

(C) 2018 by the authors. Licensee MDPI, Basel, Switzerland. This article is an open access article distributed under the terms and conditions of the Creative Commons Attribution (CC BY) license (http:/ / creativecommons.org/licenses/by/4.0/). 